\title{
Small Angle Neutron Scattering and X-Ray Diffraction Studies of Nanocrystalline Titanium Dioxide
}

\author{
Muhammad Nasir Khan, Javaid Bashir \\ Physics Division, PINSTECH, Islamabad, Pakistan \\ E-mail: nasir@pinstech.org.pk \\ Received February 28, 2011; revised April 12, 2011; accepted April 28, 2011
}

\begin{abstract}
Nanocrystalline titanium dioxide powder is characterized for phase analysis as well as particle size and its distribution by x-ray diffraction and small angle neutron scattering measurements. Analysis of the SANS data in the momentum transfer range $q=0.1-1.8 \mathrm{~nm}^{-1}$ reveals an average particle size of $24.82 \mathrm{~nm}$ in good agreement with the particle size determined earlier by transmission electron microscopy. XRD measurement proves co-existence of rutile and anatase phases in this commercial $\mathrm{TiO}_{2}$ nanocrystalline powder.
\end{abstract}

Keywords: Titanium Dioxide, Average Size, SANS, Microstructure, X-Ray Diffraction

\section{Introduction}

Microstructural aspects such as particle size and its distribution, defect structures of the materials having high technological applications are of great interest from both safety and performance perspectives. Nanophase oxide materials are interesting due to their potential and unique mechanical, chemical, electrical, optical and magnetic properties. Among various dioxides titanium dioxide $\left(\mathrm{TiO}_{2}\right)$ has been suggested as being a material that could have a series of phases with hardness possibly approaching diamond $[1,2]$. Pure as well as doped nanostructured $\mathrm{TiO}_{2}$ materials are essential for the development of advanced electrochemical solar cells, photo catalytic, electron sensitive devices and optical coatings. $\mathrm{TiO}_{2}$ in its entire known three phases is technologically important material for numerous applications. $\mathrm{TiO}_{2}$ with rutile phase has been widely used as white pigment material because of its good scattering effect that protects materials from ultraviolet light. It has also been used for optical coatings, beam splitters because of its high dielectric constant and refractive index. The material also possesses good oil adsorption ability, tinting power, and chemical stability under strong acidic or basic conditions $[3,4] . \mathrm{TiO}_{2}$ with an anatase structure has been used as a photocatalyst for photodecomposition and solar energy conversion because of its high photoactivity [5-8]. $\mathrm{TiO}_{2}$ shows different electrical characteristics with oxygen partial pressure, because it has wide chemical stability and a non-stoichiometric phase region. For this reason, it can also be used as humidity sensor and high temperature oxygen sensors $[9,10]$.

Nanocrystalline $\mathrm{TiO}_{2}$ powders are generally synthesized by different techniques such as the sulphate or chloride processes, sol-gel method, and hydrothermal synthesis [11-13]. All these synthesis techniques are vital and have advantages/disadvantages over each other but still there is a need for simple and cost effective process for the commercial production of $\mathrm{TiO}_{2}$ nano powders. For the practical applications of these high technological materials their physical and chemical properties are greatly dependent on the particle size. Therefore, it is necessary to know the true particle size, shape, and their distribution in these nanocrystalline materials. X-ray diffraction (XRD) is a powerful tool to identify the different phases of compound but small angle neutron scattering (SANS) is well suited technique for an analysis of microstructural characterization of such high technological materials, allowing for quantitative measurements of particle/pores morphology, particle size distribution. In this paper the results are demonstrated for the nanocrystalline commercial DEGUSSA P25 $\mathrm{TiO}_{2}$ for particle size determination and distribution by SANS and phase analysis by XRD. The results are being compared with earlier published data.

\section{Experimental}

The XRD pattern for $\mathrm{TiO}_{2}$ nanocrystalline powder was measured by Philips Xpert PRO diffractometer equipped 
with PW3015 generator, goniometer and X'celerator (SSD detectors) in the $2 \theta$ ranges $20^{\circ}-90^{\circ}$. The small angle neutron scattering measurement was carried out with the SANS instrument at the HANARO reactor of the Korea Atomic Energy Research Institute, Korea. The SANS instrument had a typical pinhole geometry using neutrons with wavelength of $5.08 \AA$. Wavelength spread was $(\Delta \lambda / \lambda \approx 10 \%)$. The distance from sample to detector was $3 \mathrm{~m}$. The measured $q$-value range was $0.1-1.8 \mathrm{~nm}^{-1}$. The scattering pattern of the sample was recorded with a two-dimensional position sensitive detector with an active area of $64.5 \times 64.5 \mathrm{~cm}^{2}$, and were circularly averaged to produced one-dimensional intensity profiles. The scattering intensity data were reduced to absolute scale by using standard porous silica in $1 \mathrm{~mm}$ path length quartz cell. Scattering intensities were measured from $\mathrm{TiO}_{2}$ nanocrystalline powder filled in $25 \mathrm{~mm}$ diameter quartz cell with $1 \mathrm{~mm}$ path length.

\section{Results \& Discussion}

Figure 1 shows the XRD pattern for the nanocrystalline $\mathrm{TiO}_{2}$ powder. All of the peaks on this pattern belong to rutile and anatase phases of this compound. These peaks have been marked as (A) for anatase and (R) for rutile phase respectively on the diffraction pattern. The general proportion of these phases in commercial $\mathrm{TiO}_{2}$ has been known as $75 \%$ for anatase and $25 \%$ for rutile [12]. In Figure 1, the XRD data gives this ratio as $75 \%: 25 \%$ for anatase and rutile phases on the basis of maximum peak intensities ratios for the respective two phases. No traces of others $\mathrm{TiO}_{2}$ phases such as brookite or $\mathrm{Zr}$ based srilankite were observed which often appear in most of the nanocrystalline $\mathrm{TiO}_{2}$ powders prepared by different processes [12]. The particle size in the rutile phase was reported to be larger than that of anatase by different

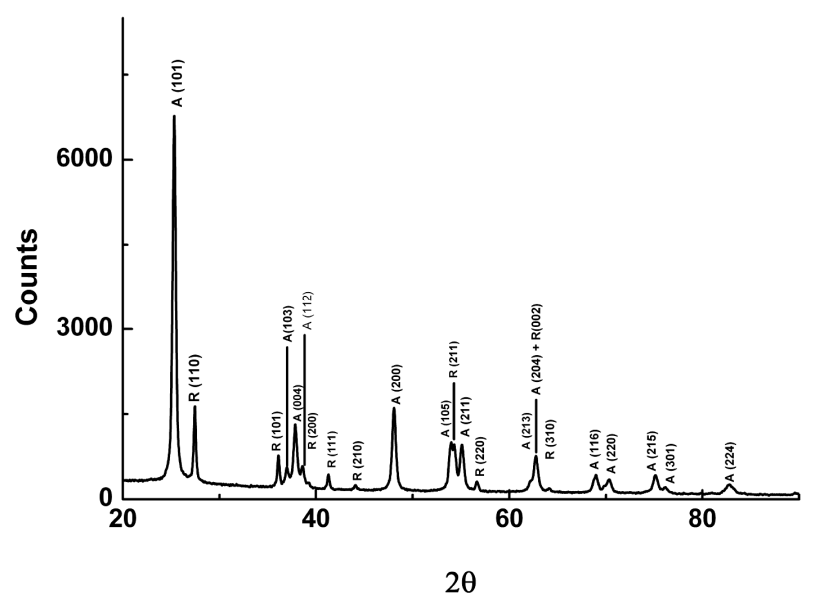

Figure 1. XRD pattern for the Degussa P25 nanocrystalline $\mathrm{TiO}_{2}$ powder. synthesis processes because of the agglomeration process, which is the growth of primary $\mathrm{TiO}_{2}$ particles with an increase of thermal energy [12]. The above finding of the phases and their ratio in this study are well in agreement with the earlier published X-ray and synchrotron X-ray diffraction data on the same material $[14,15]$.

Figure 2 shows the one-dimensional (1-D) SANS pattern for the $\mathrm{TiO}_{2}$ nanocrystalline powder circularly averaged from the 2-D measured pattern. The 2-D pattern (not shown here) clearly revealed isotropic scattered symmetric behavior, thus indicating that structures inside the powders are spherical. As the particles in the powder possess different sizes and internal structures, therefore the macroscopic differential scattering cross section for spherical particles as reported in [16] is given by;

$$
\frac{\mathrm{d} \Sigma}{\mathrm{d} \Omega}(Q)=\Delta \eta^{2} \int_{0}^{\infty}\left(\frac{4}{3} \pi R^{3}\right)^{2} N(R) F^{2}(Q, R) \mathrm{d} R
$$

where $\Delta \eta^{2}$ is the scattering contrast ( $\eta$ is the scattering length density) depending on the chemical composition of both the scattering centers and matrix, $R$ is the radius of the spherical particles. $N(R) \mathrm{d} R$ is the number of the particles per unit volume with a typical size between $R$ and $R+\mathrm{d} R . F^{2}(Q, R)$ is the form factor which in the case of spherical particles is given by;

$$
F(Q, R)=3 \frac{\sin (Q R)-Q R \cos (Q R)}{(Q R)^{3}}
$$

The scattering length density was calculated with assumption that the particles smaller than $50 \mathrm{~nm}$ are dispersed equally in this material. The model fitting of the real space size distribution, log-normal size distribution to the scattering pattern was performed using non linear least square fitting [17];

$$
N(R)=\sum_{i} N_{o i} \exp \left[-0.5\left(\frac{\ln (R)-\ln \left(R_{o i}\right)}{S_{i}}\right)^{2}\right]
$$

and

$$
\langle R\rangle=\frac{\int R N(R) \mathrm{d} R}{\int N(R) \mathrm{d} R}
$$

where $N_{o}, R_{o}$ and $S$ are the standard scaling factors, center and width parameters of this distribution type. The lognormal distribution was fitted in the $q$-range $0.1-1.1$ $\mathrm{nm}^{-1}$ in Equation (1). The results of the fitting along with the observed data are shown in Figure 2.

The average $\langle R\rangle$, of the particles calculated from the fitted parameters comes out to be $9.62 \mathrm{~nm}$ for the nanocrystalline commercial DEGUSSA P25 material as shown in Figure 3 from the number distribution function curve determined using the spherical model calculations 


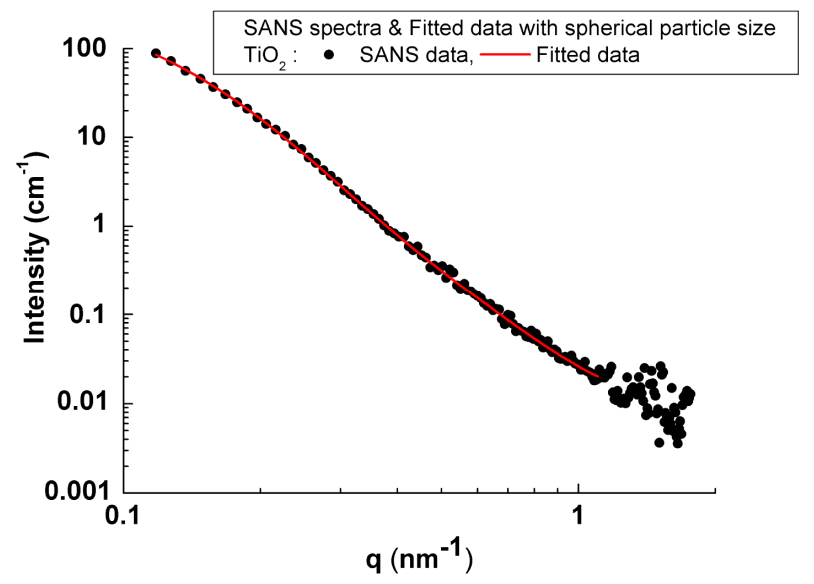

Figure 2. 1-D SANS Pattern averaged from the 2-D pattern for the nanocrystalline $\mathrm{TiO}_{2}$ powder sample; (solid lines shows spherical model fitting to the observed data).

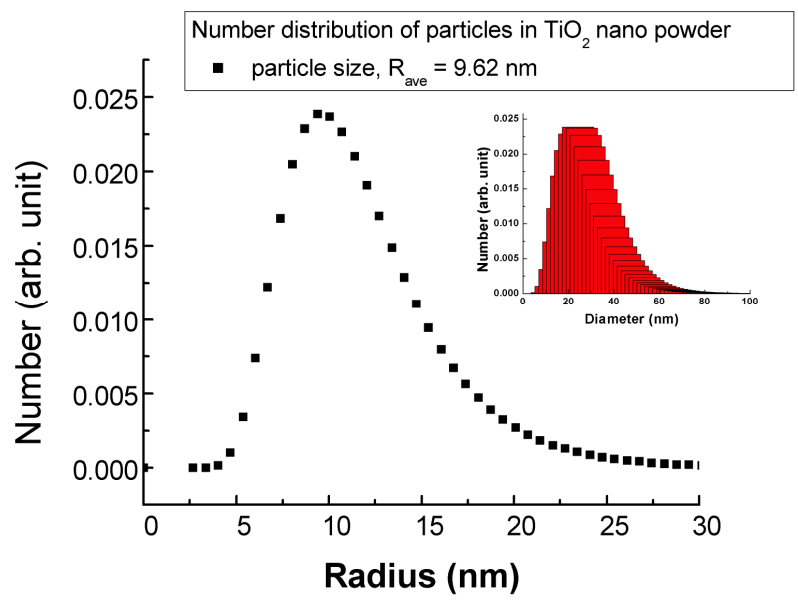

Figure 3. Particle size distribution for the nanocrystalline $\mathrm{TiO}_{2}$ powder sample (Inset shows the true particle diameter distribution).

mentioned above. Similar approached was used to estimate the size and microstructure of precipitates affected by coiling temperature in ultra low carbon steel in [18].

The size distribution was calculated in terms of the true particles diameter by the formula given below as mentioned in [19] and is shown in the inset of Figure 3.

$$
D=(5 / 3)^{\frac{1}{2}} 2 R_{g}
$$

The average diameter of the spherical particle comes out to be $24.82 \mathrm{~nm}$ from SANS results, which corresponds well with the particles size determined by SunJae Kim, et al. [20] using transmission electron microscopy technique.

\section{Conclusions}

For nanocrystalline DEGUSSA $\mathrm{P} 25, \mathrm{TiO}_{2}$ powder the
SANS results revealed that the average particle size of $24.82 \mathrm{~nm}$ agrees well with the earlier results of TEM. The XRD pattern proved coexistence of anatase and rutile phases with ratio of $75 \%$ and $25 \%$ respectively. Furthermore, the results demonstrates the power and utility of the SANS technique in characterizing microstructure, particle size, and distribution in nanocrystalline materials.

\section{Acknowledgements}

The authors are thankful to Mr. Chang-Hee Lee, B. S. Seong and Shin Enjoo of Neutron Scattering Division, KAERI, for their help in the SANS experiment and and data analysis. Their local hospitality is also highly acknowledged.

\section{References}

[1] J. M. Leger, J. Haines and B. Blanzat, "Materials Potentially Harder Than Diamond: Quenchable High-Pressure Phases of Transition Metal Oxides," Journal of Materials Science Letters, Vol. 13, 1994, pp. 1688-1690. doi:10.1007/BF00451741

[2] A. Fahmi, C. Minot, B. Silvi and M. Causa, "Theoretical Analysis of the Structures of $\mathrm{TiO}_{2}$ Crystals," Physical Review B, Vol. 47, 1993, pp. 11717-11724. doi:10.1103/PhysRevB.47.11717

[3] H. Cheng, J. Ma, Z. Zhao and L. Qi, "Hydrothermal Preparation of Uniform Nanosize Rutile and Anatase Particles," Chemistry of Materials, Vol. 7, No. 4, 1995, pp. 663-671. doi:10.1021/cm00052a010

[4] R. U. Flood and D. Fitzmaurice, "Preparation, Characterization and Potential Dependent Optical Absorption Spectroscopy of Unsupported Large Area Transparent Nanocrystalline $\mathrm{TiO}_{2}$ Membranes," Journal of the Physical Chemistry, Vol. 99, No. 22, 1995, pp. 8954-8958. doi: $10.1021 / \mathrm{j} 100022 \mathrm{a} 004$

[5] T. Fuyuki and H. Matsunami, "Electronic Properties of the Interface between $\mathrm{Si}$ and $\mathrm{Tio}_{2}$ Deposited at Very Low Temperatures," Japanese Journal of Applied Physics, Vol. 25, No. 9, 1986, pp.1288-1291. doi:10.1143/JJAP.25.1288

[6] P. V. Kamat and N. M. Dimitrijevic, "Colloidal Semiconductors as Photo-Catalyst for Solar Energy Conversion," Solar Energy, Vol. 44, 1990, pp. 83-98. doi:10.1016/0038-092X(90)90070-S

[7] S. A. Larson and J. L. Falconer, "Characterization of $\mathrm{TiO}_{2}$ Photocatalysis Used in Trichlorethene Oxidation," Applied Catalysis B: Environmental, Vol. 4, 1994, pp. 325-342. doi:10.1016/0926-3373(94)00030-1

[8] A. L. Micheli, "Fabrication and Performance Evaluation of Titania Automotive Exhaust Gas Sensor," American Ceramics Society Bulletin, Vol. 54, 1984, p. 694-698.

[9] K. L. Siefering and G. L.Griffin, "Kinetics of Low Pres- 
sure Chemical Vapor Deposition $\mathrm{TiO}_{2}$ from Titanium Tetraisopropxide," Journal of the Electrochemical Society, Vol. 137, No. 4, 1990, pp. 814-818. doi:10.1149/1.2086561

[10] H. Tang, K. Prasad, R. Sanjines and F. Levy, "TiO ${ }_{2}$ Anatase Thin Films as Gas Sensors," Sensors Actuators B, Vol. 26-27, 1995, pp. 71-75. doi:10.1016/0925-4005(94)01559-Z

[11] X. Z. Ding, Z. Z. Qi and Y. Z. He, "Effect of Hydrolysis Water on the Preparation of Nano Crystalline Titania Powder via a Sol-Gel Process," Journal of Materials Science Lettters, Vol. 14, 1995, pp. 21-22.

[12] S. J. Kim, S. D. Park, Y. H. Jeong and S. Park, "Homogeneous Precipitation of $\mathrm{TiO}_{2}$ Ultrafine Powders from Aqueous $\mathrm{TiOCl}_{2}$ Solution," Journal of American Ceramic Society, Vol. 82, No. 4, 1999, pp. 927-932. doi:10.1111/j.1151-2916.1999.tb01855.x

[13] Q. Chen, Y. Qian, Z. Chen, G. Zhou and Y. Zhang, "Preparation of $\mathrm{TiO}_{2}$ Powders with Different Morphologies by an Oxidation Hydrothermal Combination Method," Materials Letters, Vol. 22, 1995, p. 77-80. doi:10.1016/0167-577X(94)00227-4

[14] Y. F. Chen, C. Y. Lee, M. Y. Yeng and H. T. Chiu, "The Effect of Calcination Temperature on the Crystallinity of $\mathrm{TiO}_{2}$ Nanopowder," Journal of Crystal Growth, Vol. 247, 2003, pp. 363-370. doi:10.1016/S0022-0248(02)01938-3

[15] M. N. Khan, K. Shahzad and J. Bashir, "Thermal Atomic
Displacements in Nanocrystalline Tltanium Dioxide Studied by Synchrotron X-Ray Diffraction," Journal of Physics D: Applied Physics, Vol. 41, No. 8, 2008, Article ID: 085409.

[16] U. K. Srdic, V. Wiedenman, M. Winterer and H. Hahn, "Nono Sized Ceramics of Coated Alumina and Zirconia Analysed with SANS," Journal of Applied Crystallography, Vol. 33, 2000, pp. 483-487. doi:10.1107/S0021889899014727

[17] J. Kohlbrecher, "SASFITTCL (2) version 0.2 Alpha," PSI, Zurich, 1999.

[18] E. J. Shin, B. S. Seong, Y. S. Han, K. P. Hong, C. H. Lee and H. J. Kang, "Effect of Precipitate Size and Dispersion on Crystallization Behaviour in Ti-Added Ultra Low Carbon Steel," Journal of Applied Crystallography, Vol. 36, No. 3, 2003, pp. 624-628. doi:10.1107/S0021889803003856

[19] J. Blasing, P. Kohlert, M. Zacharias and P. Veit, "X-Ray Fine Structure Investigations of Germanium Nanoclusters," Journal of Applied Crystallography, Vol. 31, 1998, pp. 589-593. doi:10.1107/S0021889898001071

[20] S.-J. Kim, J.-K. Lee, H.-G. Lee, S.-J. Kim and K.-S. Lee, "Photocatalytic Properties of Rutile $\mathrm{Tio}_{2}$ Acicular Particles in Aqueous 4-Chlorophenol Solution," Journal of Materials Research, Vol. 18, No. 3, 2003, pp. 729-732. doi:10.1557/JMR.2003.0098 